\title{
Targeting CA 19-9 with a humanized monoclonal antibody at the time of surgery may decrease recurrence rates for patients undergoing resections for pancreatic cancer, cholangiocarcinoma and metastatic colorectal cancer
}

\author{
Shreya Gupta, James D. McDonald, Reed I. Ayabe, Tahsin M. Khan, Lauren A. Gamble, Surajit Sinha, \\ Cathleen Hannah, Andrew M. Blakely, Jeremy L. Davis, Jonathan M. Hernandez \\ Surgical Oncology Program, Center for Cancer Research, National Cancer Institute, Bethesda, MD, USA \\ Correspondence to: Jonathan M. Hernandez, MD. 10 Center Drive, Rm 4-3742, Bethesda, MD 20892, USA. Email: jonathan.hernandez@nih.gov.
}

\begin{abstract}
Operable gastrointestinal cancers continue to pose significant challenges. Radical resections are rarely curative, and chemotherapy is able to reduce tumor recurrence for only a small percentage of patients. Despite the obvious advantages of extirpation of the identifiable tumor(s), the inflammatory milieu that accompanies surgery and the obligate time off cytotoxic agents allows for activation of remote quiescent disseminated tumor cells, leading to metastatic recurrence. We are conducting a study to determine the safety and efficacy of immediate peri-operative MVT-5873, a cytotoxic monoclonal antibody targeting carbohydrate antigen 19-9 (CA 19-9), in patients undergoing resections pancreatic cancer, cholangiocarcinoma or metastatic colorectal cancer to the liver. Eligible patients will receive a single dose of MVT-5873 three days before resection and four post-operative infusions, before beginning standard adjuvant regimens. MVT-5873 is a human IgG1 antibody isolated from a patient following immunization with a sLe ${ }^{2}-$ KLH vaccine. MVT-5873 demonstrated cell surface binding in sLe $e^{a}$ positive human tumor lines and has been shown to be potent in complement-dependent cytotoxicity assays and antibody-dependent cell mediated cytotoxicity assays. In patients with metastatic CA 19-9 producing pancreatic adenocarcinoma, MVT-5873 treatment has been shown to decrease serum CA 19-9 levels and prevent tumor progression. The use of perioperative MVT-5873 has the potential to reduce recurrence rates and prolong survival after resection. This trial may open the door for investigation of additional and/or synergistic agents in the immediate perioperative period and usher in a new paradigm in the management of surgically treated cancers.
\end{abstract}

Trial registration: https://clinicaltrials.gov/ct2/show/NCT03801915?term=MVT\&rank=3.

Keywords: carbohydrate antigen 19-9 (CA 19-9); adjuvant therapy; pancreatic cancer; cholangiocarcinoma; liver metastasis

Submitted Oct 16, 2019. Accepted for publication Dec 17, 2019.

doi: 10.21037 /jgo.2020.02.01

View this article at: http://dx.doi.org/10.21037/jgo.2020.02.01

\section{Introduction/study rationale}

The "Achilles heel" of surgically treated cancers is recurrence following resection. A growing body of literature supports the notion that metastatic spread occurs very early in the process of tumorigenesis, even before radiologic detection has occurred. Seeding of distant organs by metastatic cells is thought to be followed by a period of quiescence and subsequent outgrowth leading to the development of overt metastases. Admittedly however, the biology of micrometastases remains a "black box" for which there appears to be no immediate avenues for interrogation, at least at present. Nonetheless, we believe that the identification and targeting of the mechanisms that support the survival and outgrowth of latent, disseminated tumor cells holds 
great promise for the prevention of tumor recurrence. This belief stems from the incontrovertible reality that, although resection remains the only chance for prolonged overall survival (OS) and potential cure for patients with resectable cancers of the gastrointestinal tract, this end-point is infrequently achieved secondary to recurrence of disease at a distant site. For example, analysis of randomized prospective trial data and single institution series strongly suggests that nearly half of all patients undergoing resections for pancreatic cancer, cholangiocarcinoma and metastatic colorectal cancer to the liver will experience a recurrence of disease in the first 12-15 months following removal of tumor(s) (1-3). In other words, these patients underwent an operation for likely abbreviated benefit in terms of survival.

It has been postulated that the operation itself may trigger growth of micrometastases by inciting an inflammatory milieu and triggering an angiogenic switch in remote disseminated tumor cells. Substantial data exist in mice supporting this concept (4). Moreover, it is known that surgery is associated with an increase in circulating tumor cells (CTC) (5). We hypothesize that the administration of an agent directed against tumor cells, if substantial circulating concentrations at the time of resection can be achieved, will lead to mitigation of any "pro-tumor" effects of the resection itself with subsequent abrogation of early tumor recurrences. Unfortunately, chemotherapy has deleterious effects on wound healing that makes its administration in the immediate peri-operative period an extremely high-risk endeavor. However, a well-tolerated monoclonal antibody targeting a tumor cell epitope holds great promise in mitigating early recurrences and thereby increasing the efficacy of resection for cancers of the pancreas, liver and bile ducts without adversely affecting recovery and outcomes from surgery. This concept of immediate peri-operative therapy is unique and additive to what is generally undertaken with high-risk cancers. For example, patients could receive standard neo-adjuvant and/ or adjuvant therapies in addition to immediate peri-operative therapy without altering the standard regimens. In short, the employment of immediate peri-operative therapy, if demonstrated to be safe, could add an additional tool in the oncologist's armamentarium for resectable high-risk cancers.

The Sialyl Lewis A (sLe ${ }^{a}$ ) antigen is an epitope present on carbohydrate antigen 19-9 (CA 19-9), a well-known plasma tumor marker overexpressed on the surface of many epithelial tumors $(6,7) . s \mathrm{se}^{\mathrm{a}}$ is an oligosaccharide expressed primarily as a proteoglycan that acts as a ligand for $\mathrm{E}$ selectin, facilitating tumor cell adhesion and extravasation (8).
On normal cells, expression of CA 19-9 is restricted to the apical brush border, a relatively inaccessible location due to the basement membrane, and the tight junctions of mature and polarized ductal epithelium (9). Comparatively, CA 19-9 is widely expressed on tumors of the gastrointestinal tract, with high expression seen in up to $94 \%$ of pancreatic cancers as well as bile duct carcinomas, and to a lesser degree in colorectal carcinoma (10). Given the limited expression in non-cancerous tissue and high expression rate of CA 19-9 in patients with gastrointestinal tract cancers, MVT-5873 (which targets the sLe epitope of CA 19-9) was developed as an antibody-based biopharmaceutical and selected for immediate peri-operative use in targeting micrometastases.

MVT-5873 is a fully human derived IgG1 lambda antibody that was identified from blood lymphocytes from a breast cancer patient following immunization with a sLe ${ }^{a}-K L H$ vaccine (11). This vaccine has been shown to induce high titers of both $\operatorname{IgG}$ and $\operatorname{IgM}$ antibodies against $\mathrm{sLe}^{\mathrm{a}}$ in mice and humans without cross-reactivity to other similar blood group carbohydrate antigens (11). Evaluation of the specificity of MVT-5873 demonstrated cell surface binding in sLe $\mathrm{a}^{\mathrm{a}}$ positive colon cancer (HT29 and Colo205), ovarian cancer (SW626), small cell lung cancer (DMS79), and pancreatic cancer $(\mathrm{BxPC} 3)$ cell lines, but not to a sLe ${ }^{a}$ negative melanoma (SK-MEL28) cell line (12). Importantly, MVT-5873 did not bind to Lea, sLex, Ley, or other related carbohydrates when evaluated by enzymelinked immunosorbent assay (ELISA) and surface plasmon resonance (12). In addition, a glycan array with 465 distinct carbohydrates revealed that MVT-5873 had exquisite specificity for the carbohydrate epitope of the $\mathrm{sLe}^{\mathrm{a}}$ antigen. MVT-5873 has been shown to be potent in complementdependent cytotoxicity (CDC) assays and antibodydependent cell mediated cytotoxicity (ADCC) assays. Both mechanisms are consistent with antitumor activity.

\section{Methods/trial design}

\section{Design}

This is a prospective, Phase II trial designed to determine the efficacy and safety of peri-operative MVT-5873 for subjects with operable pancreatic, liver and bile duct cancers with elevated CA 19-9 levels. All patients will be enrolled at the NIH Clinical Center in Bethesda, Maryland (NCT03801915). This study is supported by the Intramural Research Program of the NIH, National 


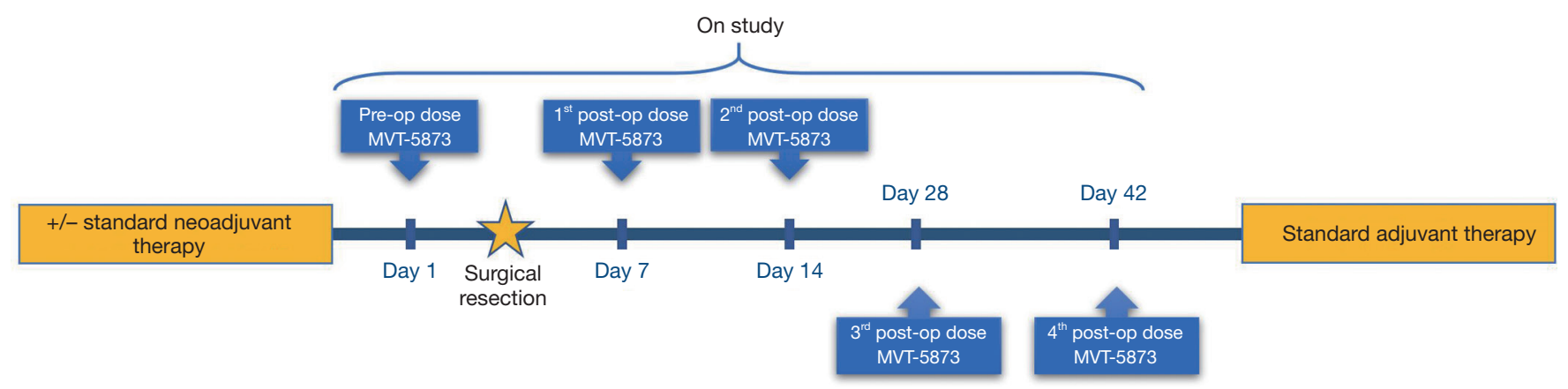

Figure 1 MVT-5873 administration timeline in the immediate peri-operative period.

Cancer Institute (NCI). Subjects will be recruited with the following histologies: metastatic colorectal cancer $(\mathrm{N}=24)$, cholangiocarcinoma $(\mathrm{N}=24)$ and pancreatic cancer $(\mathrm{N}=23)$.

\section{Eligibility}

Eligible patients must be 18 years or older, have an ECOG performance status of $<2$ and a histologically or cytologically confirmed diagnosis of adenocarcinoma of the pancreas, bile duct or metastatic colorectal cancer to the liver. Disease must be resectable with a standard pancreatectomy (pancreaticoduodenectomy or distal pancreatectomy) or liver/bile duct resection (combined resection with the primary tumor for patients with metastatic colorectal cancer is permitted). Patients may have received neoadjuvant therapy. Eligible patients must have serum CA 19-9 elevations greater than the upper limit of normal but less than 2,500 U/mL. Furthermore, the following laboratory values are screened for inclusion.

* Leukocytes: >3,000/ mcL;

* Absolute neutrophil count: 1,500/mcL;

* Platelets: >90,000/mcL;

* Creatinine: $<1.5 \times$ ULN;

* Total bilirubin: $<10 \times$ ULN (periampullary);

* Total bilirubin: $<2.5 \times$ ULN (liver);

* Total bilirubin: $<1.5 \times$ ULN (distal pancreas);

* AST/ALT: $<5 \times$ ULN (periampullary);

* AST/ALT: $<5 \times$ ULN (liver);

* AST/ALT: $<2 \times$ ULN (distal pancreas).

\section{Exclusion}

Exclusion criteria includes the presence of disease outside the confines of a standard operation for patients with periampullary cancers, and the presence of disease outside the liver for patients with intrahepatic/hilar cholangiocarcinoma or metastatic colorectal cancer (other than the primary colorectal tumor). Patients who are on other clinical trials or investigational drugs are excluded. Subjects who are fewer than 28 days from any other systemic agent are also excluded. Pregnant women, or women suspected to be pregnant are excluded given the unknown effects on fetus. Chronic systemic illness, other active malignancies, active hepatitis $\mathrm{B}$ or $\mathrm{C}$, or long-term infectious disease are also exclusionary.

\section{Intervention}

Eligible patients will receive a pre-operative dose of MVT5873 three days prior to the planned operation to remove all demonstrable disease. MVT-5873 will be given as an infusion over 120 minutes. Following the operation, patients will receive a total of four doses of MVT-5873; the first two doses on postoperative days 4 and 11, which we anticipate will occur prior to discharge. The third dose will be administered on the normally scheduled postoperative clinic visit and followed by a final dose one month after discharge from the hospital and prior to the start of adjuvant treatment (Figure 1).

\section{Study endpoints}

The primary endpoints for this study are to determine the safety and efficacy of immediate perioperative use of MVT5873 for patients undergoing a standard surgical resection for pancreatic adenocarcinoma, cholangiocarcinoma or metastatic colorectal cancer to the liver. Safety will be determined based on timely surgical resection (hepatic resection or pancreatic resection) following administration of first dose of MVT-5873. Safety will also be assessed 
by perioperative morbidity including procedure related complications (e.g., pancreatic leak or hepatic insufficiency) as compared to recent randomized trials for the respective procedures. We defined efficacy as an increase in 1-year disease-free survival (DFS) rates by $50 \%$ as compared to that documented in the literature per histology.

\section{Scientific endpoints}

As outlined in the introduction, micrometastases are the unmeasurable, unidentifiable cells that result in recurrence at a site distant from the primary tumor. We aim to target these cells with the application of MVT-5873. When a patient on study undergoes a liver operation, we have developed techniques to sustain that resected tumor-bearing liver, which contains both macro and micrometastatic cells, outside of that patient for extended periods of time. This provides for an unparalleled opportunity to interrogate and define the heretofore undefinable-micrometastatic cells in a target organ. We envision that macroscopic tumor biology (from the tumor being resected) will be compared to microscopic tumor biology (from the disseminated micrometastases in the ostensibly normal portions of liver) enabling design or selection of appropriate adjuvant agents. Moreover, these agents can be trialed ex vivo in subsequent livers without the concern for patient toxicities, enabling refinements, titrations and combinations not previously possible.

\section{Discussion and conclusions}

The current paradigms of treatment for localized gastrointestinal cancers involve resection with cytotoxic chemotherapy, applied either before and/or afterwards. However, early recurrence patterns are commonly seen in certain high-risk patients, including those with pancreatic cancer, cholangiocarcinoma and those with colorectal cancer that has already spread to the liver. These recurrences are the result of tumor cells that have already seeded the target organs (liver, lung, etc.). With this study, we seek to target these cells at a time when they are likely to become activated secondary to stress and inflammatory signals that obligatorily accompany an operation. If the application of a monoclonal antibody to a tumor-epitope proves to be safe and effectively mitigates early recurrences when given in the immediate peri-operative period (this is the time period when systemic agents are routinely held), we hypothesize that a new era of agent application will ensue with the aim of increasing the efficacy of surgery as applied to cancers.

\section{Acknowledgments}

Funding: National Cancer Institute/Center for Cancer Research, 10 Center Drive, Building 10, Bethesda, MD 20892, USA.

\section{Footnote}

Conflicts of Interest: The authors have no conflicts of interest to declare.

Ethical Statement: The authors are accountable for all aspects of the work in ensuring that questions related to the accuracy or integrity of any part of the work are appropriately investigated and resolved.

Open Access Statement: This is an Open Access article distributed in accordance with the Creative Commons Attribution-NonCommercial-NoDerivs 4.0 International License (CC BY-NC-ND 4.0), which permits the noncommercial replication and distribution of the article with the strict proviso that no changes or edits are made and the original work is properly cited (including links to both the formal publication through the relevant DOI and the license). See: https://creativecommons.org/licenses/by-nc$\mathrm{nd} / 4.0 /$.

\section{References}

1. D'Angelica M, Kornprat P, Gonen M, et al. Effect on outcome of recurrence patterns after hepatectomy for colorectal metastases. Ann Surg Oncol 2011;18:1096-103.

2. Doussot A, Gonen M, Wiggers JK, et al. Recurrence Patterns and Disease-Free Survival after Resection of Intrahepatic Cholangiocarcinoma: Preoperative and Postoperative Prognostic Models. J Am Coll Surg 2016;223:493-505.e2.

3. Neoptolemos JP, Palmer DH, Ghaneh P, et al. Comparison of adjuvant gemcitabine and capecitabine with gemcitabine monotherapy in patients with resected pancreatic cancer (ESPAC-4): a multicentre, open-label, randomised, phase 3 trial. Lancet 2017;389:1011-24.

4. Rachman-Tzemah C, Zaffryar-Eilot S, Grossman M, et al. Blocking Surgically Induced Lysyl Oxidase 
Activity Reduces the Risk of Lung Metastases. Cell Rep 2017;19:774-84.

5. Arnoletti JP, Zhu X, Almodovar AJ, et al. Portal Venous Blood Circulation Supports Immunosuppressive Environment and Pancreatic Cancer Circulating Tumor Cell Activation. Pancreas 2017;46:116-23.

6. Magnani JL, Nilsson B, Brockhaus M, et al. A monoclonal antibody-defined antigen associated with gastrointestinal cancer is a ganglioside containing sialylated lacto-Nfucopentaose II. J Biol Chem 1982;257:14365-9.

7. Magnani JLS, Koprowski H, Ginsburg V. Identification of the gastrointestinal and pancreatic cancer-associated antigen detected by monoclonal antibody 19-9 in the sera of patients as a mucin. Cancer Res 1983;43:5489-92.

8. Sato M, Narita T, Kimura N, et al. The association of sialyl Lewis(a) antigen with the metastatic potential of human

Cite this article as: Gupta S, McDonald JD, Ayabe RI, Khan TM, Gamble LA, Sinha S, Hannah C, Blakely AM, Davis JL, Hernandez JM. Targeting CA 19-9 with a humanized monoclonal antibody at the time of surgery may decrease recurrence rates for patients undergoing resections for pancreatic cancer, cholangiocarcinoma and metastatic colorectal cancer. J Gastrointest Oncol 2020;11(2):231-235. doi: 10.21037/ jgo.2020.02.01 colon cancer cells. Anticancer Res 1997;17:3505-11.

9. Koprowski H, Steplewski Z, Mitchell K, et al. Colorectal carcinoma antigens detected by hybridoma antibodies. Somatic Cell Genet 1979;5:957-71.

10. Passerini R, Cassatella MC, Boveri S, et al. The pitfalls of CA19-9: routine testing and comparison of two automated immunoassays in a reference oncology center. Am J Clin Pathol 2012;138:281-7.

11. Ragupathi G, Damani P, Srivastava G, et al. Synthesis of sialyl Lewis(a) (sLe (a), CA19-9) and construction of an immunogenic sLe(a) vaccine. Cancer Immunol Immunother 2009;58:1397-405.

12. Sawada R, Sun SM, Wu X, et al. Human monoclonal antibodies to sialyl-Lewis (CA19.9) with potent CDC, ADCC, and antitumor activity. Clin Cancer Res 2011;17:1024-32. 